\title{
ON THE DIFFERENTIABILITY \\ OF LIPSCHITZ-BESOV FUNCTIONS
}

\author{
JOSÉ R. DORRONSORO
}

\begin{abstract}
L^{r}$ and ordinary differentiability is proved for functions in the Lipschitz-Besov spaces $B_{a}^{p, q}, 1 \leq p<\infty, 1 \leq q \leq \infty, a>0$, using certain maximal operators measuring smoothness. These techniques allow also the study of lacunary directional differentiability and of tangential convergence of Poisson integrals.
\end{abstract}

1. Introduction. The differentiability properties of functions in the Sobolev spaces $L_{k}^{p}, 1 \leq p \leq \infty, k \in \mathbf{N}$, (i.e., $L^{p}$ functions $f$ whose weak partials of order $k$ are also in $L^{p}$ ) are very well known. For instance, if $k>n / p$ and $f \in L_{k}^{p}, f$ has a.e. an ordinary differential of order $k$; that is, for a.e. $x \in \mathbf{R}^{n} f(x+y)-P_{k} f(y, x)=$ $o\left(|y|^{k}\right)$, with $P_{k} f$ the Taylor polynomial of $f$ at $x$,

$$
P_{k} f(y, x)=\sum_{|J| \leq k} \frac{f_{J}(x) y^{J}}{J !},
$$

where for each $n$-index $J=\left(j_{i}, \ldots, j_{n}\right) \in \mathbf{N}^{n}, f_{J}$ denotes the $J$ th order weak partial of $f$.

If $k<n / p$ only $L^{p^{*}}$ differentiability is possible, $p^{*}=n p / n-a p ;$ more precisely, if $f \in L_{k}^{p}, f$ has a $k$ th order $L^{p^{*}}$ differential (or a $\left(p^{*}, k\right)$ differential) a.e.; that is, for a.e. $x$

$$
\left(f_{|y| \leq t} \mid f\left(x+y-\left.P_{k} f(y, x)\right|^{p^{*}} d y\right)^{1 / p^{*}}=o\left(t^{k}\right),\right.
$$

where $f_{E} f$ denotes the mean $\int_{E} f d x /|E|$. Finally, if $k=n / p, p^{*}=\infty$, and any $f \in L_{n / p}^{p}$ has an $(r, k)$ differential a.e. for all $r<\infty$.

Of course, functions in $L_{k}^{p}$ have also lower order differentials and for them the exceptional set becomes smaller. In fact, denoting by $B_{a, q}$ the Bessel capacity associated with $L_{a}^{q}$ (see [My] for its definition), given $m \in \mathbf{N}$ with $0 \leq m<k$, any $f \in L_{k}^{p}$ has $B_{k-m, p}$ a.e. a $\left(p^{*}, m\right)$ differential if $k<n / p$, an $(r, m)$ differential for any $r<\infty$ if $k=n / p$, and an $(\infty, m)$ differential (i.e., an $m$ th order ordinary differential) if $k>n / p$ (if $k-m>n / p$ by $B_{k-m, p}$ a.e. we mean everywhere).

Starting with the work of Calderón and Zygmund $[\mathbf{C Z}]$, these results and the corresponding ones for the Bessel potential spaces $L_{a}^{p}=\left\{f=J_{a} g: g \in L^{p}\right\}$, $J_{a}$ being the Bessel potential operator, $a>0,1<p<\infty$, have been studied by several authors, among them C. P. Calderón, Fabes and Riviere [CFR] and

Received by the editors September 10, 1986.

1980 Mathematics Subject Classification (1985 Revision). Primary 46E35, 26A16; Secondary 42B25.

Supported by C.A.I.C.Y.T. 2805-83. 
T. Bagby, Deignan, Federer and Ziemer $[\mathbf{B Z}, \mathbf{D Z}, \mathbf{F Z}]$. The purpose of this paper is to deal with these questions in the context of the Lipschitz-Besov spaces $B_{a}^{p q}$, $a>0,1 \leq p<\infty, 1 \leq q \leq \infty$, of those $L^{p}$ functions $f$ such that

$$
\int_{\mathbf{R}^{n}}|y|^{-n-a q}\left\|\Delta_{y}^{k} f(\cdot)\right\|_{p}^{q} d y=|f|_{a, p, q},
$$

where $\Delta_{y} f(x)=f(x+y)-f(x)$ and $k=[a]+1$; with the norm $\|f\|_{a, p, q}=$ $\|f\|_{p}+|f|_{a, p, q}, B_{a}^{p q}$ becomes a Banach space (see [St] or [T] for more properties of $\left.B_{a}^{p q}\right)$. For an integer $k$, we have the embeddings $B_{k}^{p q} \subset L_{k}^{p}$, with $1 \leq q \leq p$ if $p \leq 2$ and $1 \leq q \leq 2$ if $p>2$ [St, Chapter V] which inmediately give $k$ th order differentiability results for these ranges of $q$. In general, these results cannot be improved: for instance, the one variable function

$$
f(x)=g(x)\left(\sum_{1}^{\infty} 2^{-n} n^{-1 / 2} \cos 2^{n} x\right)
$$

with $g \in C_{0}^{\infty}, g=1$ if $|x| \leq 1, g=0$ if $|x| \geq 2$, belongs to $B_{1}^{p q}, p \geq 2, q>2$ but is differentiable only in a zero measure set [Z, pp. 47 and 206].

Therefore, we will concentrate in the study of lower order differentials, for which the full range $1 \leq q \leq \infty$ can be considered. In this context partial results have been given by D. Adams [Ad1], Neugebauer [N1, N2], and Stocke [Sto], and all follow the pattern set by the $L_{a}^{p}$ cases. In fact, if $a>n / p$ one should expect ordinary differentiability to hold, whereas if $a \leq n / p$, because of Herz's imbedding theorem, only $L^{r}$ differentiability, $r<p^{*}$, is to be expected in the full range $1 \leq q \leq \infty$, while $L^{p^{*}}$ differentiability should hold for restricted values of $q$. Also, we can consider a capacity type set function $A_{a, p, q}$ associated to $B_{a}^{p q}$ (more precisely, a family $A_{a, p, q}^{s}, 0<s<a$, of set functions, all having the same zero sets; see $\S 2$ ), and the exceptional sets should be measured in terms of the corresponding Besov capacities. We postpone their definition until $\S 2$, and state now our main results.

THEOREM 1. Given $f \in B_{a}^{p q}, a>0,1 \leq p<\infty, 1 \leq q \leq \infty$, and a real number $b$ with $0 \leq b<a$, then

(i) if $a \leq n / p$, for any $r<p^{*} f$ has an $(r, b)$ differential $A_{a-b, p, q}$ a.e.;

(ii) if $a>n / p, f$ has an $(\infty, b)$ differential $A_{a-b, p, q}$ a.e. (if $a-b>n / p, A_{a-b, p, q}$ a.e. is to be interpreted as e.e.)

(For nonintegral $b$, we mean by $(r, b)$ differentiability that

$$
\left(f_{|y| \leq t}\left|f(x+y)-P_{b} f(y, x)\right|^{r} d y\right)^{1 / r}=o\left(t^{b}\right)
$$

where $P_{b} f$ denotes the $[b]$ th order Taylor polynomial of $f$ at $x$; for $(\infty, b)$ differentials just change the $L^{r}$ norm to an $L^{\infty}$ norm).

As mentioned above, although this result is quite satisfactory if $a>n / p$, one should have $p^{*}$ differentiability for certain $q$ 's when $a \leq n / p$. This is indeed the case if $1 \leq q \leq p$, but the measure of the exceptional set is then less precise, because of the following fact: if $q \geq p$ any set of zero $H^{n-a p}$ Hausdorff measure has also zero $A_{a, p, q}$ capacity [Ad1], but this is not presently known to hold of $1 \leq q<p$ (see however [Ad2]); we are thus forced to introduce another set function, the 
bracket $\left[H^{n-a p}, A_{a, p, q}\right]$ (see [Ad1, N1] and $\S 2$ ); as we shall see, if $H^{n-a p}\left(E_{1}\right)=$ $A_{a, p, q}\left(E_{2}\right)=0$, then $\left[H^{n-a p}, A_{a, p, q}\right]\left(E_{1} \cup E_{2}\right)=0$. We now have the following theorem.

THEOREM 2. If $f \in B_{a}^{p q}, 1 \leq p<\infty, a \leq n / p, 1 \leq q \leq p$ and $b$ with $0 \leq b<a$ is given, $f$ has a $\left(p^{*}, b\right)$ differential $\left[H^{n-(a-b)} p, A_{a-b, p, q}\right]$ a.e.

Note that any $f \in B_{a}^{p p}$ has then a $\left(p^{*}, b\right)$ differential $A_{a-b, p, p}$ a.e. (and hence $B_{a-b, p}$ a.e.; see [Ad 2]).

The proofs of these theorems rely on estimates for certain maximal operators first introduced by A. P. Calderón and Scott $[\mathbf{C S}]$ and extensively studied by DeVore and Sharpley [DVS]. They can also be used to study lacunar directional differentiability of Lipschitz functions. More precisely, given a point $u \in S_{n-1}$, the unit sphere in $\mathbf{R}^{n}$, we will say that $f$ has lacunar differential of order $b$ in the direction $u$ at a point $x \in \mathbf{R}^{n}$ if

$$
\lim _{k \rightarrow \infty} 2^{k b}\left|f\left(x+2^{-k} u\right)-P_{b} f\left(2^{-k} u, x\right)\right|=0 .
$$

If $f \in L_{a}^{p}$, C. Calderón [CC2] has recently proved the existence $B_{a-b, p}$ a.e. of a lacunary differential of order $b$ in the direction of each $u \in S_{n-1}$ (note that such an $f$ can be essentially unbounded in any arbitrarily small ball). In the Lipschitz case we have

THEOREM 3. If $f \in B_{a}^{p q}, a \leq n / p, 1<p<\infty, 0 \leq b<a$, and $u \in S_{n-1}, f$ has $A_{a-b, p, q}$ a.e. a lacunar differential of order $b$ in the direction of $u$.

Another application of these techniques is to study tangential convergence of Poisson integrals of functions in $B_{a}^{p q}$. For potential spaces these type of results are due to Nagel, Rudin, Shapiro and Stein [NRS, NS], and Mizuta [Mz] has studied the case of $B_{a}^{p p}$. In [Do] it is shown how tangential convergence can be related to the regularity of the functions involved, and here we have

THEOREM 4. If $x \in \mathbf{R}^{n}, c>0$ and $1<r<\infty$, consider the set $D_{c, r}(x)=$ $\left\{(x, y): z \in \mathbf{R}^{n}, y>0,|x-z| \leq y^{1-c r / n}\right\}$. Then:

(i) If $1 \leq p<\infty, 1 \leq q \leq \infty, a \leq n / p$ and $b$ is such that $0 \leq b<a$, the Poisson integral $u(z, y)$ of any $f \in B_{a}^{p q}$ converges to $f(x)$ inside $D_{b, s}(x)$ for $A_{a-b, p, q}$ a.a. $x \in \mathbf{R}^{n}$ and any $s<p$.

(ii) If moreover, $1 \leq q \leq p, u(z, y)$ converges to $f(x)$ inside $D_{b, p}(x)$ for $\left[H^{n-(a-b) p}, A_{a-b, p, q}\right]$ a.a. $x \in \mathbf{R}^{n}$.

(Observe that if $a>n / p$, any $f \in B_{a}^{p q}$ is continuous; also, if $a=n / p, 1 \leq q \leq p$, convergence inside regions with exponential contact holds for functions in $B_{a}^{p q}$; see [Mz, Do].)

This paper is organized as follows. $\S 2$ contains the definition and some properties of Besov capacities, and the Calderón-Scott-DeVore-Sharpley maximal operators are discussed in $\S 3$. Theorems 1 and 2 are proved in $\S 4$ and Theorems 3 and 4 in $\S \S 5$ and 6 respectively.

2. Besov capacities. It is well known (see [St, p. 153]) that the Bessel potential operator $J_{b}$ is an isomorphism between $B_{a}^{p q}$ and $B_{a+b}^{p q}, 1 \leq p, q \leq \infty, 0<a, b$. Following [N1, Sto], this isomorphism allows us to define capacity type set functions: given $a>0,1 \leq p, q \leq \infty$, for any $s$ with $0<s<a$ and $E \subset \mathbf{R}^{n}$, 
we set

$$
A_{a, p, q}^{s}(E)=\inf \left\{\|g\|_{s, p, q}^{p}: g \geq 0, J_{a-s} g \geq 1_{E}\right\},
$$

where $1_{E}$ stands for the characteristic function of $E$. We first show that the $a, p, q$ Besov capacity of a set is essentially independent of $s$.

LEMMA. If $0<s<s^{\prime}<a$, there is a constant $C=C_{s, s^{\prime}}$, such that for any $E \subset \mathbf{R}^{n}$

$$
A_{a, p, q}^{s}(E) / C \leq A_{a, p, q}^{s^{\prime}}(E) \leq C A_{a, p, q}^{s}(E) .
$$

PROOF. If $g \in B_{p, q}^{s}, g \geq 0$ and $J_{a-s} g \geq 1_{E}, h=J_{s^{\prime}-s} g$ is also $\geq 0, J_{a-s^{\prime}} h \geq 1_{E}$ and $\|h\|_{s^{\prime}, p, q} \leq C\|g\|_{s, p, q}$; thus $A_{a, p, a}^{s^{\prime}}(E) \leq A_{a, p, q}^{s}(E)$, Conversely, if $0<e<$ $\min (1, s)$ and $h \in B_{p, q}^{s^{\prime}}, h \geq 0, J_{a-s^{\prime}} h \geq 1_{E}$, consider $g=J_{s-e}(|v|)$, where $v$ is such that $J_{s^{\prime}-e} v=h$; then $g \geq 0, J_{a-s} g=J_{a-s^{\prime}}\left(J_{s^{\prime}-e}(|v|)\right) \geq J_{a-s^{\prime}} h \geq 1_{E}$ and $\|g\|_{s, p, q} \leq C\|h\|_{s, p, q}$. Therefore, $A_{a, p, q}^{s}(E) \leq C A_{a, p, q}^{s^{\prime}}(E)$.

As a consequence, all the $A_{a, p, q}^{s}$ capacities have the same zero sets; in this situation we will just write $A_{a, p, q}(E)=0$ or $A_{a, p, q}$ a.e. without specifying any $s$ index.

For a given $E \subset \mathbf{R}^{n}$ we define

$$
\left[H^{n-a p}, A_{a, p, q}^{s}\right](E)=\inf \left\{H^{n-a p}\left(E_{1}\right)+A_{a, p, q}^{s}\left(E_{2}\right): E=E_{1} \cup E_{2}\right\} ;
$$

as a consequence of the lemma all the $\left[H^{n-a p}, A_{a, p, q}^{s}\right]$ are equivalent set functions and in particular all have the same zero sets. In this case we will also write $\left[H^{n-a p}, A_{a, p, q}\right](E)=0$, without specifying the $s$ parameter. $C$,

If $1<p<\infty$, the embeddings $L_{a+e}^{p} \subset B_{a}^{p q} \subset L_{a-e}^{p}, e>0$, imply that for some

$$
B_{a-e, p}(E) / C \leq A_{a, p, q}^{s}(E) \leq C B_{a+e, p}(E)
$$

in particular, if $A_{a, p, q}(E)=0, E$ has $n-a p$ Hausdorff dimension. When $p=1$, the embeddings [DVS] $B_{a}^{1, q} \subset B_{a-e}^{11} \subset L_{a-n / r^{\prime}-2 e^{\prime}}^{r}$ with $r<n / n-a$, imply [Ad1] that $H_{\infty}^{n-a+e}(E) \leq C A_{a, 1, q}^{s}(E)$ for any $e>0$, where if $0 \leq b<n, 0<d \leq \infty$,

$$
H_{d}^{n-b}(E)=\inf \left\{\sum_{i}\left|Q_{i}\right|^{1-b / n}: E \subset \bigcup Q_{i}, Q_{i} \text { cubes, side } Q_{i} \leq d\right\}
$$

moreover, if $a<n$, an easy homogeneity argument [Sto] gives $A_{a, 1, q}^{s}(Q(x, r)) \leq$ $C r^{n-a} A_{a, 1, q}^{s}(Q(1,0))(Q(x, r)=$ cube of center $x$ and side $2 r)$, if $0<r<1$; this and the countable subadditivity of $A_{a, 1, q}^{s}$ imply that $A_{a, 1, q}^{s}(E) \leq C H_{1}^{n-a}(E)$.

Another way of defining Besov capacities is by setting for a compact $K$

$$
A_{a, p, q}^{\prime}(K)=\inf \left\{\|f\|_{a, p, q}^{p}: f \in C_{0}^{\infty}, f \geq 1_{K}\right\}
$$

and extending it as an outer capacity to general sets in $\mathbf{R}^{n}$ (see [Ad1, Sto]; replacing the $B_{a}^{p q}$ norm with the $L_{a}^{p}$ norm we obtain the usual Bessel capacity). It can then be proved [Sto, Lemma 1] that $A_{a, p, q}^{s}(E)=0$ implies $A_{a, p, q}^{\prime}(E)=0$, $1<p, q<\infty$.

We finally note that functions in $B_{a}^{p q}$ are well defined $A_{a, p, q}$ a.e.; in fact, if $0<s<\min (1, a)$ and $g \in B_{s}^{p q}$, the integral $\int J_{a-s}(x-y) g(y) d y$ is finite for $A_{a, p, q}$ a.a. $x \in \mathbf{R}^{n}[\mathbf{N 1}$, Sto], and as a consequence of the dominated convergence theorem, $\int_{|y| \leq t} J_{a-s}(y) g(x+y) d y$ tends to 0 with $t$ for $A_{a, p, q}$ a.a. $x$. 
3. Maximal operators. If $a>0, m=[a], f \in L^{p}, 1 \leq p \leq \infty$, and $Q$ is a cube in $\mathbf{R}^{n}$, we denote by $P_{Q} f$ the unique polynomial in $\mathbf{P}_{m}=$ ppolynomials of degree $\leq m\}$ such that for any multi-index $J=\left(j_{1}, \ldots, j_{n}\right) \in \mathbf{N}^{n}$ with $|J|=j_{1}+\cdots+j_{n} \leq$ $m, \int_{Q}\left(f(y)-P_{Q} f(y)\right) y^{J} d y=0$.

$P_{q} f$ is a best approximation polynomial in the sense that [DVS, p. 17]

$$
\inf \left\{f_{Q}|f-P| d y: P \in \mathbf{P}_{m}\right\} \sim f_{Q}\left|f-P_{Q} f\right| d y
$$

$(A \sim B$ means $A / C \leq B \leq C A$ for some universal constant $C$ ); moreover [DVS, p. 17], if $D^{J}=\left(\partial / \partial x_{1}\right)^{j_{1}} \cdots\left(\partial / \partial x_{n}\right)^{j_{n}}$,

$$
\sup \left\{\left|D^{J} P_{Q} f(z)\right|: z \in Q\right\} \leq C|Q|^{-|J| / n} f_{Q}|f| .
$$

Setting for $t>0$

$$
E f(x, t)=\sup \left\{f_{Q}\left|f-P_{Q} f\right|: x \in Q, \text { side } Q=t\right\},
$$

$C_{a}^{p}$ is then defined [DVS] as the space of those $f \in L^{p}$ such that $G_{a} f(x)=$ $\sup \left\{t^{-a} E f(x, t): t>0\right\}$ is also in $L^{p}$; with the norm $\|f\|_{a, p}=\|f\|_{p}+\left\|G_{a} f\right\|_{p}$, $C_{a}^{p}$ becomes a Banach space.

Now, if $x \in Q^{\prime} \subset Q$, with $Q, Q^{\prime}$ having side lengths $t, t^{\prime}$, and we write

$$
P_{Q} f(y)=\sum_{|J| \leq m} C_{J}(Q)(y-x)^{J} / J !
$$

and $P_{Q^{\prime}} f(y)$ in a similar manner, we have for any $|J| \leq m[$ Do]

$$
\left|C_{J}(Q)-C_{J}\left(Q^{\prime}\right)\right| \leq C \int_{t^{\prime}}^{t} E f(x, s) s^{-|J|} \frac{d s}{s} ;
$$

there estimates can be further refined.

Proposition 1. With $x, Q, Q^{\prime}, t, t^{\prime}$ as before, if $G_{a} f \in L^{p}$ and $a-|J|<n$,

$$
\begin{aligned}
\left|C_{J}(Q)-C_{J}\left(Q^{\prime}\right)\right| \leq & C\left(t^{\prime}\right)^{a-|J|-n} \int_{|z| \leq t^{\prime}} G_{a} f(x+z, t) d z \\
& +C \int_{t^{\prime} \leq|z| \leq t}|z|^{a-|J|-n} G_{a} f(x+z, t) d z,
\end{aligned}
$$

where $G_{a} f(x, t)=\sup \left\{s^{-a} E f(x, s): 0<s \leq t\right\}$; also, if $a-|J|=n$,

$$
\begin{aligned}
\left|C_{J}(Q)-C_{J}\left(Q^{\prime}\right)\right| \leq & C \log \frac{t}{t^{\prime}} \int_{|z| \leq t^{\prime}} G_{a} f(x+z, t) d z \\
& +C \int_{t^{\prime} \leq|z| \leq t} G_{a} f(x+z, t) \log \frac{t}{|z|} d z .
\end{aligned}
$$

Finally, if $a-|J|>n / p$, and $s$ is such that $a-|J|>n / s \geq n / p$,

$$
\left|C_{J}\left(Q^{\prime}\right)-C_{J}(Q)\right| \leq C t^{a-|J|-n / s}\left(\int_{|z| \leq t} G_{a} f(x+z, t)^{s} d z\right)^{1 / s} .
$$


Proof. Denoting by $B(x, s)$ the ball centered at $x$ with radius, (2) implies

$$
\begin{aligned}
\left|C_{J}\left(Q^{\prime}\right)-C_{J}(Q)\right| & \leq C \int_{t^{\prime}}^{t}\left(f_{B(x, s)} E f(z, s) d z\right) s^{-|J|} \frac{d s}{s} \\
& \leq C \int_{t^{\prime}}^{t} s^{a-|J|-n} \int_{B(x, s)} G_{a} f(z, t) d z d s \\
& \leq C \int_{t^{\prime}}^{t} s^{a-|J|-n}\left(\int_{0}^{s} r^{n-1} \int_{S_{n-1}} G_{a} f(x+r u, t) d u d r\right) \frac{d s}{s}
\end{aligned}
$$

and (3) and (4) now follow by Fubini's theorem. To prove (5), just note that (2) implies

$$
\begin{aligned}
\left|C_{J}(Q)-C_{J}\left(Q^{\prime}\right)\right| & \leq C \int_{0}^{t} r^{a-|J|}\left(f_{B(x, r)} G_{a} f(z, r)^{s}\right)^{1 / s} \frac{d r}{r} \\
& \leq C t^{a-|J|}\left(f_{B(x, t)} G_{a} f(z, t)^{s} d z\right)^{1 / s} .
\end{aligned}
$$

We observe next that $G_{a}$ can be seen as a derivative.

PROPOSITION 2. If $f \in B_{a}^{p q}, a>0,1 \leq p, q \leq \infty$ and $0<e<\min (1, a)$, there is an $F \in B_{e}^{p q}$ with $\|F\|_{e, p, q} \leq\|f\|_{a, p, q}$ such that $G_{a}-e f(x, 1) \leq C F(x)$.

PROOF. Writing $a^{\prime}=a-e$ and $f=J_{a^{\prime}} g, g \in B_{e}^{p q}$, and $k=\left[a^{\prime}\right]$, we then have [Do, Theorem 4]

$$
\begin{aligned}
E f(x, t) & \leq C t^{a^{\prime}} E g(x, t)+C t^{k+1} \int_{t}^{\infty} s^{a^{\prime}-k-1} E g(x, s) \frac{d s}{s} \\
& \leq C t^{a^{\prime}} m g(x)+C t^{a^{\prime}} \int_{1}^{\infty} s^{a^{\prime}-k-1} 1_{s} * g(x) \frac{d s}{s},
\end{aligned}
$$

where $m$ denotes the "local" maximal function

$$
m g(x)=\sup \left\{f_{Q}|g| d z: x \in Q,|Q| \leq 1\right\}
$$

and $1_{s}(z)=s^{-n} 1_{Q(0,1)}(z / s)$.

Writing now $g=J_{e-d} h, h \in B_{d}^{p q}$, and taking into account that for side $Q \leq 1$, $f_{Q} J_{d}(x+z) d z \leq C J_{d}(x)$ [AS, p. 418], it follows that $m g \leq C J_{e-d}(|h|$ ). Also, $\Delta_{y}\left(1_{s} * g\right)(x)=1_{s} *\left(\Delta_{y} g\right)(x)$, and

$$
\begin{aligned}
\left\|\Delta_{y}\left(\int_{1}^{\infty} s^{a^{\prime}-k-1} 1_{s} * g(\cdot) \frac{d s}{s}\right)\right\|_{p} & \leq \int_{1}^{\infty} s^{a^{\prime}-k-1}\left\|1_{s} * \Delta_{y} g\right\|_{p} \frac{d s}{s} \\
& \leq C\left\|\Delta_{y} g\right\|_{p}
\end{aligned}
$$

thus, taking $e<1$, it follows that $\int_{1}^{\infty} s^{a^{\prime}-k-1} 1_{s} * g(x) d s / s$ is in $B_{e}^{p q}$.

We can derive now the existence of Taylor polynomials of degree $[b]$ of a given $f \in B_{a}^{p q}$ for any $b$ with $0 \leq b<a$. 
Since $B_{a}^{p q} \subset C_{a}^{p}$, for any $a^{\prime}=a-e<a, G_{a^{\prime}} f \in L^{p}$; thus, if $|J| \leq b$ and $a-|J| \leq n / p,(3)$ and the local estimates for Bessel potentials imply

$$
\left|C_{J}(Q)-C_{J}\left(Q^{\prime}\right)\right| \leq C \int_{|z| \leq t} J_{a^{\prime}-|J|}(z) F(x+z) d z,
$$

with $F$ the $B_{e}^{p q}$ function of Proposition 2. Therefore, as $t$ tends to $0, C_{J}(Q)$ converges $A_{a-|J|, p, q}$ a.e. to a finite limit $f_{J}(x)$ satisfying

$$
\left|f_{J}(x)-C_{J}(Q)\right| \leq C \int_{|z| \leq t} J_{a^{\prime}-|J|}(z) F(x+z) d z .
$$

Moreover, if $a-|J|>n / p$ and $e, s$ are chosen so that $a^{\prime}-|J|>n / s \geq n / p,(5)$ implies that as $t$ tends to $0 C_{J}(Q)$ converges e.e. to a finite limit $f_{J}(x)$ satisfying

$$
\left|f_{J}(x)-C_{J}(Q)\right| \leq C t^{a^{\prime}-|J|}\left(f_{|z| \leq t} F(x+z)^{s} d s\right)^{1 / s} .
$$

If $|J|=0, C_{0}(Q)=P_{Q} f(x)$ tends to $f(x)$ a.e. [DVS, p. 9], and thus, redefining $f$ in a measure 0 set, (7) and (8) can be rewritten as the $A_{a, p, q}$ a.e. estimates

$$
\left|f(x)-P_{Q} f(x)\right| \leq C \int_{|z| \leq t} J_{a^{\prime}}(z) F(x+z) d z
$$

$$
\left|f(x)-P_{Q} f(x)\right| \leq C t^{a^{\prime}-n / s}\left(\int_{|z| \leq t} F(x+z)^{s} d z\right)^{1 / s} ;
$$

according to whether $a^{\prime} \leq n / p$ or $a^{\prime}>n / s \geq n / p$.

Finally, it follows from the above that if $0 \leq b<a$ the Taylor polynomial of degree $\leq b$ of an $f \in B_{a}^{p q}$,

$$
P f(y, x)=\sum_{|J| \leq b} \frac{f_{J}(x) y^{J}}{J !}
$$

is defined for $A_{a-b, p, q}$ a.a. $x \in \mathbf{R}^{n}$ (in fact, $f_{J}$ coincides with the $J$ th order weak partial of $f$ ).

4. Differentiability of Lipschitz functions. We now prove Theorem 1. If $b$ is such that $0 \leq b<a$, writing $Q=Q(x, t), x \in \mathbf{R}^{n}, t>0$, we have

$$
\begin{aligned}
& t^{-b}\left|f(x+y)-P_{b} f(y, z)\right| \leq t^{-b}\left|f(x+y)-P_{Q} f(x+y)\right| \\
&+C t^{-b} \sum_{|J| \leq b}\left|f_{J}(x)-C_{J}(Q)\right| t^{|J|} \\
&+C t^{-b} \sum_{b<|J|}\left|C_{J}(Q)\right| t^{|J|} \\
& \leq \mathrm{I}(y, x, t)+\mathrm{II}(x, t)+\mathrm{III}(x, t) .
\end{aligned}
$$

Setting $f=J_{a-e} g, g \in B_{e}^{p q}$, with $e<1$ and $a-e>b$, suppose first $a-b \leq n / p$; using either (7) or (8) depending on whether $a-|J| \leq n / p$ or $a-|J|>n / p$, 
we estimate II as

$$
\begin{aligned}
\mathrm{II}(x, t) \leq & C \sum_{\substack{|J| \leq b \\
a-|J|>n / p}} t^{|J|-b} t^{a-|J|-e}\left(f_{|z| \leq t} F(x+z)^{s} d z\right)^{1 / s} \\
& +C \sum_{\substack{|J| \leq b \\
a-|J| \leq n / p}} t^{|J|-b} \int_{|z| \leq t} J_{a-e-|J|}(z) F(x+z) d z
\end{aligned}
$$

(the first sum may be empty; for instance, if $a \leq n / p$ ); thus

$$
\begin{aligned}
\mathrm{II}(x, t) \leq & C\left(t^{a-b-e}\left(f_{|z| \leq t} F(x+z)^{s} d z\right)^{1 / s}\right. \\
& \left.+\int_{|z| \leq t} J_{a-e-b}(z) F(z+z) d z\right) \\
\leq & C \int_{|z| \leq t} J_{a-e-b}(z) m_{s}(F)(x+z) d z
\end{aligned}
$$

where $m_{s}(F)=\left(m\left(|F|^{s}\right)\right)^{1 / s}$, which if $1 \leq s<p$ is again a function in $B_{e}^{p q}[\mathbf{N} 1]$, and if $p=s=1$, just as we did in Proposition 2, can be bounded by $C G$, where $G$ is another $B_{e}^{p q}$ function. In either case $\operatorname{II}(x, t)$ tends to $0 A_{a-b, p, q}$ a.e. as $t$ goes to 0 . When $a-b>n / p$, the situation is simpler: we just use (6) to obtain

$$
\mathrm{II}(x, t) \leq C \sum_{|J| \leq b} t^{|J|-b} t^{a-|J|-e-n / p}\|F\|_{p}=C\|F\|_{p} t^{a-b-e-n / p},
$$

which, if $a-b-e>n / p$, tends to 0 e.e.

Turning our attention to III, we write for $T>t Q(x, T)$ as $Q^{*}$; then

$$
\begin{aligned}
\operatorname{III}(x, t) \leq & \left.C \sum_{\substack{|J| \leq b \\
a-|J|>n / p}}+\sum_{\substack{|J| \leq b \\
a-|J| \leq n / p}}\right) t^{|J|-b}\left|C_{J}(Q)-C_{J}\left(Q^{*}\right)\right| \\
& +C \sum_{|J|>b} t^{|J|-b}\left|C_{J}\left(Q^{*}\right)\right| ;
\end{aligned}
$$

by (1), the second term is bounded by

$$
C\|f\|_{p} \sum_{|J|>b} t^{|J|-b} T^{-|J|-n / p}
$$

whereas in the first one the first sum is empty if $a-b \leq n / p$, and in any case, using either (5) or (4) and Proposition 2, it can be bounded by

$$
\begin{aligned}
& C t^{a-b-e}\left(f_{Q^{*}} F(z)^{s} d z\right)^{1 / s} \\
& \quad+C \sum_{\substack{|J| \leq b \\
a-|J| \leq n / p}} t^{|J|-b} \int_{t \leq|z| \leq T}|z|^{a-|J|-e-n} F(x+z) d z .
\end{aligned}
$$


We therefore have

$$
\begin{aligned}
\operatorname{III}(x, t) \leq & C t^{a-b-e}\left(\int_{Q^{*}} F(x+z)^{s} d z\right)^{1 / s}+C \int_{Q^{*}}|z|^{a-b-e-n} F(x+z) d z \\
& +C\|f\|_{p} \sum_{|J|>b} t^{|J|-b} T^{-|J|-n / p} \\
= & A(x, t)+B(x, T)+C(t, T) ;
\end{aligned}
$$

here $A$ and $B$ tend to 0 with $T A_{a-b, p, q}$ a.e. if $a-b \leq n / p$ and e.e. if $a-b>n / p$; thus, given any $\delta>0$, if $x$ is such a point, we can find a $T_{\delta}$ such that for all $t<T_{\delta}$ $A(x, t)+B\left(x, T_{\delta}\right)<\delta$, and we can find now a $t_{\delta}$ small enough so that $C\left(t, T_{\delta}\right)<\delta$ for all $t<t_{\delta}$. Hence $\operatorname{III}(x, t)$ also tends to $0 A_{a-b, p, q}$ a.e.

Finally we deal with I. If $a \leq n / p$, we can write any $r<n p / n-a p$ as $1 / r=$ $1 / s+1 / u-1$, with $s<p$ and $u<n / n-a$; choosing $e$ small enough so that $u<n / n-(a-e),(9)$ and Young's inequality imply

$$
\begin{aligned}
\left(f_{|y| \leq t} I(y, x, t)^{r} d y\right)^{1 / r} & \leq C t^{-b-n / r} t^{a-e-n+n / u}\left(\int_{|z| \leq 2 t} F(x+z)^{s} d z\right)^{1 / s} \\
& =C t^{a-e-b}\left(f_{|z| \leq 2 t} F(x+z)^{s} d z\right)^{1 / s} \\
& \leq C \int_{|z| \leq 2 t} J_{a-e-b}(z) m_{s} F(x+z) d z
\end{aligned}
$$

which tends to $0 A_{a-b, p, q}$ a.e. Next, if $a>n / p$ it follows from (10) that if $|y| \leq t$,

$$
\mathrm{I}(y, x, t) \leq C t^{a-b-e}\left(f_{|z| \leq 2 t} F(x+z)^{s} d z\right)^{1 / s}
$$

provided $a-e>n / s>n / p$, and it also tends to $0 A_{a-b, p, q}$ a.e.; the theorem is thus proved.

PROOF OF THEOREM 2. If $1 \leq q \leq p$ we have the embedding $B_{a}^{p q} \subset C_{a}^{p}$ [DVS, p. 58] and, as a consequence, if $f \in B_{a}^{p q}, G_{a} f \in L^{p}$; thus estimating as before

$$
t^{-b}\left|f(x+y)-P_{b} f(y, x)\right| \leq \mathrm{I}(y, x, t)+\mathrm{II}(x, t)+\operatorname{III}(x, t)
$$

and writing for $a<n / p, p^{*}=n p / n-a p,(9)$ and Sobolev's inequality if $p>1$ give

$$
\left(f_{|y| \leq t} \mathrm{I}(y, x, t)^{p^{*}} d y\right)^{1 / p^{*}} \leq C t^{a-b}\left(f_{|z| \leq 2 t} G_{a} f(x+z)^{p} d z\right)^{1 / p}
$$

which as it is well known tends to $0 H^{n-(a-b) p}$ a.e. When $p=1$ the same argument works once we use instead of (9) the estimate

$$
\left|f(x)-P_{Q} f(x)\right|^{r} \leq C \int_{|z| \leq t}|z|^{a r-n} G_{a} f(x+z)^{r} d z
$$

$0<r \leq 1$, proved in [Do, Theorem 5]. If we estimate $\operatorname{II}(x, t)$ and $\operatorname{III}(x, t)$ as before it follows that $f$ has a $\left(p^{*}, b\right)$ derivative $\left[H^{n-(a-b) p}, A_{a-b, p, q}\right]$ a.e. 
It may be worth noting that if $f \in C_{a}^{p}$ the reasoning in Theorems 1 and 2 can be easily modified to obtain $\left(p^{*}, b\right),(r, b), r<\infty$, or $(\infty, b)$ differentiability (depending on whether $a<n / p,=n / p$ or $>n / p$ ) for $f B_{a-b . p}$ a.e. if $b$ is an integer and $H^{n-(a-b) p}$ a.e. for nonintegral $b$. Since $L_{a}^{p} \subset C_{a}^{p}$ this implies the $L_{a}^{p}$ results mentioned in the Introduction, and also differentiability for functions in the TriebelLizorkin spaces $F_{a}^{p q}, 1 \leq p, q<\infty$ (see [Tr] for their definition). We can associate with these spaces certain capacity type set functions and one could guess that the above differentiability results should be given with respect to them. This is not the case however, for D. Adams has recently shown [Ad2] that the $F_{a}^{p q}$ capacities, $1<p, q<\infty$, are all equivalent with $B_{a, p}$ (for $C_{a}^{p}$ capacities this is proved in [Do]).

5. Lacunar differentiability. Here we prove Theorem 3 . With $u \in S_{n-1}$, we estimate as before $2^{k b}\left|f\left(x+2^{-k} u\right)-P_{b} f\left(2^{-k} u, x\right)\right|$ by $\mathrm{I}\left(2^{-k} u, x, 2^{-k}\right)+\mathrm{II}\left(x, 2^{-k}\right)+$ $\operatorname{III}\left(x, 2^{-k}\right)$, and we consider only the first one, the other two going to 0 for $A_{a-b, p, q}$ a.a. $x$. With $F \in B_{e}^{p q}$ related to $f$ as before, $e$ small enough, we have again

$$
\begin{aligned}
\mathrm{I}\left(2^{-k} u, x, 2^{-k}\right) & \leq C 2^{k b} \int_{\left|y-2^{k} u\right| \leq 2^{-k}}\left|y-2^{-k} u\right|^{a-e-n} F(x+y) d y \\
& =C 2^{k b} \int_{|z| \leq 2^{-k}} F\left(x+2^{-k} u+z\right)|z|^{a-b-e-n}\left(\int_{|z| / 4 \leq|z-y| \leq|z| / 2}|z-y|^{b-n} d y\right) d z \\
& \leq C 2^{k b} \int_{|z| \leq 2 \cdot 2^{-k}} F\left(x+2^{-k} u+z\right)|z|^{a-b-e-n}\left(\int_{3|z| / 4 \leq|y| \leq 3|z| / 2}|z-y|^{b-n} d y\right) d z \\
& \leq C 2^{k b} \int_{|y| \leq 4 \cdot 2^{-k}} \int_{2|y| / 3 \leq|z| \leq 4|y| / 3} F\left(x+2^{-k} u+z\right)|z|^{a-b-e-n}|z-y|^{b-n} d z d y \\
& \leq C 2^{k b} \int_{|y| \leq 4 \cdot 2^{-k}}|y|^{a-b-e-n} \int_{|y-z| \leq 3|y|} F\left(x+2^{-k} u+z\right)|z-y|^{b-n} d z d y \\
& \leq C \int_{|y| \leq 4 \cdot 2^{-k}}^{|y|^{a-b-e-n} T(F)(x+y) d y}
\end{aligned}
$$

where if $h \geq 0$,

$$
\begin{aligned}
T h(\zeta) & =\sup _{k \geq 0} 2^{k b} \int_{|v| \leq 12 \cdot 2^{-k}}|v|^{b-n} h\left(\varsigma+2^{-k} u+v\right) d v \\
& =\sup 2^{k b} \int_{\left|w-2^{-k} u\right| \leq 12 \cdot 2^{-k}}\left|w-2^{-k} u\right|^{b-n} h(\varsigma+w) d w \\
& =\sup 2^{k n} \int K\left(2^{k} w\right) h(\varsigma+w) d w
\end{aligned}
$$

with $K(z)=|z-u|^{b-n}$ if $|z-u| \leq 12$, and zero otherwise. The kernel $K$ belongs to $L \log ^{+} L$ on its support and therefore Lemmas 1.3 and 1.4 of [CC1] imply that $T$ is bounded in $L^{p}, p>1$, and, in fact, in $B_{e}^{p q}$, for we have $|T f(x+y)-T f(x)| \leq$ $T\left(\Delta_{y} f\right)(x)$. Therefore,

$$
I\left(2^{-k} u, x, 2^{-k}\right) \leq C \int_{|y| \leq 4 \cdot 2^{-k}}|y|^{a-b-e-n} T(F)(x+y) d y
$$

which tends to 0 as $k$ goes to $\infty A_{a-b, p, q}$ a.e. 
6. Tangential boundary values. We recall first that the complement of the Lebesgue set of a function in $B_{a}^{p q}$ has zero $A_{a, p, q}$ capacity. Thus, since nontangential convergence holds on each point of the Lebesgue set, the case $b=0$ of Theorem 4 is proved.

Fix next an $f \in B_{a}^{p q}, b$ such that $0<b<a$, and $x \in \mathbf{R}^{n}$. If $(z, y) \in D_{b, s}(x)$, $s<p$, we have for $Y=y^{1-b s / n}$

$$
\begin{aligned}
|u(z, y)-f(x)| & \leq \int|f(u)-f(x)| P_{y}(z-u) d y \\
& =\int_{|x-u| \leq 2 Y}+\int_{|x-u|>2 Y}=\mathrm{I}+\mathrm{II} ;
\end{aligned}
$$

since $|z-x| \leq Y,|z-u| \geq|x-u|-|x-z| \geq|x-u| / 2$, and

$$
\mathrm{II} \leq \int|f(u)-f(x)| P_{y}\left(\frac{x-u}{2}\right) d u=2^{n} \int|f(u)-f(x)| P_{2 y}(x-u) d u
$$

which tends to 0 for all $x$ in the Lebesgue set of $f$, that is, $A_{a, p, q}$ a.e. Next, if $r=n s / n-b s$,

$$
\begin{aligned}
\mathrm{I} \leq & \int_{|x-u| \leq 2 Y}\left|f(u)-P_{b} f(u-x, x)\right| P_{y}(u-x) d u \\
& +C \sum_{0<|J| \leq b}\left|f_{J}(x)\right| \int_{|x-u| \leq 2 Y}|x-u|^{|J|} P_{y}(x-u) d u \\
\leq & C\left\|P_{y}\right\|_{r^{\prime}}\left(\int_{|x-u| \leq 2 Y}\left|f(u)-P_{b} f(u-x, x)\right|^{r} d u\right)^{1 / r} \\
& +C \sum_{0<|J| \leq b}\left|f_{J}(x)\right| Y^{|J|} .
\end{aligned}
$$

Since $f_{J}(x), 0 \leq|J| \leq b$, is finite for $A_{a-b, p, q}$ a.a. $x$, the second term tends to 0 $A_{a-b, p, q}$ a.e. The first term can be estimated by

$$
\begin{gathered}
C y^{-n / r} Y^{b+n / r} Y^{-b}\left(f_{|u| \leq 2 Y}\left|f(x+u)-P_{b} f(u, x)\right|^{r}\right)^{1 / r} \\
\leq C Y^{-b}\left(f_{|u| \leq 2 Y}\left|f(x+u)-P_{b} f(u, x)\right|^{r}\right)^{1 / r},
\end{gathered}
$$

for $Y=y^{s / r}$, and it tends to $0 A_{a-b, p, q}$ a.e. by Theorem 1 . This proves Theorem 4 in the range $1 \leq q \leq \infty$; if $1 \leq q \leq p$ we proceed in just the same way, using now Theorem 2 to obtain convergence inside $D_{b, p}(x)$ for $\left[H^{n-(a-b) p}, A_{a-b, p, q}\right]$ a.a. $x \in \mathbf{R}^{n}$.

ACKNOWLEDGMENT. The author wants to thank Professor D. Adams for letting him see unpublished work.

\section{REFERENCES}

[Ad1] D. Adams, Lectures on $L^{p}$ potential theory, Umeà Univ. Reports, No. 2, 1981.

[Ad2] _ The classification problem for the capacities associated with the Besov and Triebel-Lizorkin spaces (preprint). 
[AS] N. Aronszajn and K. T. Smith, Theory of Bessel potentials. I, Ann. Inst. Fourier (Grenoble) 11 (1961), 385-475.

[BZ] T. Bagby and W. Ziemer, Pointwise differentiability and absolute continuity, Trans. Amer. Math. Soc. 191 (1974), 129-148.

[CS] A. P. Calderón and R. Scott, Sobolev type inequalities for $p>0$, Studia Math. 62 (1978), 75-92.

[CZ] A. P. Calderón and A. Zygmund, Local properties of solutions of elliptic partial differential equations, Studia Math. 20 (1961), 171-225.

[CC1] C. Calderón, Lacunary spherical means, Illinois J. Math. 23 (1979), 476-486.

[CC2] - Lacunary differentiability of functions in $\mathbf{R}^{n}$, J. Approx. Theory 40 (1984), 148-154.

[CFR] C. P. Calderón, E. Fabes and N. Riviere, Maximal smoothing operators, Indiana Univ. Math. J. 23 (1974), 889-897.

[DZ] D. Deignan and W. Ziemer, Strong differentiability properties of Bessel potentials, Trans. Amer. Math. Soc. 225 (1977), 113-122.

[DVS] R. Devore and R. Sharpley, Maximal operators and smoothness, Mem. Amer. Math. Soc. No. 293, 1984.

[Do] J. Dorronsoro, Poisson integrals of regular functions, Trans. Amer. Math. Soc. 297 (1986), 669-685.

[FZ] H. Federer and W. Ziemer, The Lebesgue set of a function whose distribution derivatives are p-th power summable, Indiana Univ. Math. J. 22 (1972), 139-158.

[My] N. G. Meyers, A theory of capacities for potentials of functions in Lebesgue spaces, Math. Scand. 26 (1970), 255-292.

[Mz] Y. Mizuta, On the boundary limits of harmonic functions with gradient in $L^{p}$, Ann. Inst. Fourier (Grenoble) 34 (1984), 99-109.

[NRS] A. Nagel, W. Rudin and J. Shapiro, Tangential boundary behavior of functions in Dirichlet type spaces, Ann. of Math. (2) 116 (1982), 331-360.

[NS] A. Nagel and E. M. Stein, On certain maximal functions and approach regions, Adv. Math. 54 (1984), 83-106.

[N1] C. Neugebauer, Strong differentiability of Lipschitz functions, Trans. Amer. Math. Soc. 240 (1978), 295-306.

[N2] _ , Smoothness of Bessel potentials and Lipschitz functions, Indiana Univ. Math. J. 26 (1977), 585-591.

[St] E. M. Stein, Singular integrals and differentiability properties of functions, Princeton Univ. Press, Princeton, N.J., 1970.

[Sto] B. Stocke, Lifferentiability properties of Bessel potentials and Besov functions, Ark. Math. 22 (1984), 269-286.

[T] M. Taibleson, On the theory of Lipschitz spaces of distributions on Euclidean $n$-space. I, J. Math. Mech. 13 (1964), 407-479.

[Tr] H. Triebel, Theory of function spaces, Birkhäuser, 1983.

[Z] A. Zygmund, Trigonometric series, Cambridge Univ. Press, 1959.

División de Matemáticas, Facultad de Ciencias, Universidad Autónoma, 28049 MADRID, SPAIN 